\title{
Multisymplectic geometry, covariant Hamiltonians, and water waves
}

\author{
BY JERROLD E. MARSDEN \\ Control and Dynamical Systems 107-81, California Institute of Technology, \\ Pasadena, CA 91125 \\ e-mail: marsden@cds.caltech.edu \\ AND STEVE SHKOLLER \\ Center for Nonlinear Studies, Los Alamos National Laboratory, MS-B258, \\ Los Alamos, NM 87545 \\ e-mail: shkoller@cnls.lanl.gov
}

(Received 24 March 1997; revised 1 September 1997)

\begin{abstract}
This paper concerns the development and application of the multisymplectic Lagrangian and Hamiltonian formalism for nonlinear partial differential equations. This theory generalizes and unifies the classical Hamiltonian formalism of particle mechanics as well as the many pre-symplectic 2 -forms used by Bridges. In this theory, solutions of a partial differential equation are sections of a fibre bundle $Y$ over a base manifold $X$ of dimension $n+1$, typically taken to be spacetime. Given a connection on $Y$, a covariant Hamiltonian density $\mathscr{H}$ is then intrinsically defined on the primary constraint manifold $P_{\mathscr{L}}$, the image of the multisymplectic version of the Legendre transformation. One views $P_{\mathscr{L}}$ as a subbundle of $J^{1}(Y)^{\star}$, the affine dual of $J^{1}(Y)$, the first jet bundle of $Y$. A canonical multisymplectic $(n+2)$-form $\Omega_{\mathscr{H}}$ is then defined, from which we obtain a multisymplectic Hamiltonian system of differential equations that is equivalent to both the original partial differential equation as well as the Euler-Lagrange equations of the corresponding Lagrangian. Furthermore, we show that the $n+12$-forms $\omega^{(\mu)}$ defined by Bridges are a particular coordinate representation for a single multisymplectic $(n+2)$-form and, in the presence of symmetries, can be assembled into $\Omega_{\mathscr{H}}$. A generalized Hamiltonian Noether theory is then constructed which relates the action of the symmetry groups lifted to $P_{\mathscr{L}}$ with the conservation laws of the system. These conservation laws are defined by our generalized Noether's theorem which recovers the vanishing of the divergence of the vector of $n+1$ distinct momentum mappings defined by Bridges and, when applied to water waves, recovers Whitham's conservation of wave action. In our view, the multisymplectic structure provides the natural setting for studying dispersive wave propagation problems, particularly the instability of water waves, as discovered by Bridges. After developing the theory, we show its utility in the study of periodic pattern formation and wave instability.
\end{abstract}




\section{Introduction}

The canonical symplectic structure on the cotangent bundle of a given configuration space provides a natural correspondence between Hamiltonian vector fields that govern the evolution of conservative ordinary differential equations and the Hamiltonian functions which describe them. The setting of tangent and cotangent bundles also provides a natural setting for the Lagrangian description of dynamics and the Legendre transformation that connects the Lagrangian and Hamiltonian points of view.

In either case, the use of vector fields for the description of the dynamics is natural, because for ordinary differential equations there is a single distinguished variable, time. On the contrary, in systems of partial differential equations solutions depend on multiple variables, usually spatial as well as temporal, so one can make the case that a single vector field is not the appropriate point of view because it would require collapsing all of the spatial structure of a solution to a single point of phase space. This occurs when a choice is made to consider the time coordinate separately and describe the dynamics in terms of an infinite-dimensional space of fields at a given instant in time. Although this methodology has been very successful, availing itself to the powerful organizing structure of the theory of evolution operators from a point of view of functional analysis, its immediate affect is a break of manifest covariance.

To maintain a covariant description one can use a generalization of symplectic geometry known as multisymplectic geometry. This subject has a long and distinguished history that we shall not review in this article; rather, we follow the framework established in [7-9], wherein relativistic field theories with Dirac-Bergmann type constraints are considered in a Lagrangian formalism while the Hamiltonian formalism relies on a 'space + time' (or 3+1) split. These references contain citations to much of the important literature and history of the subject.

It is interesting that the structure of connection is not necessary to intrinsically define the Lagrangian formalism (as shown in the preceding references), while for the intrinsic definition of a covariant Hamiltonian the introduction of such a structure is essential. Of course, one can avoid a connection if one is willing to confine ones attention to local coordinates. We give an intrinsic definition of the covariant Hamiltonian so that we may examine the fundamental interplay of the equivariance of Hamiltonian and Lagrangian densities with respect to group actions.

Our objective is to use a variant of the multisymplectic Hamiltonian formalism to generalize and make intrinsic the seminal and extremely important work of Bridges [1-3] on wave propagation, periodic pattern formation and linear instability. Roughly speaking, our main result states that in the case of $n$ distinct and possibly unbounded spatial directions, the $n+1$ pre-symplectic 2 -forms introduced by Bridges are actually contained in a single higher degree multisymplectic $(n+2)$-form and that, in the presence of symmetries, these many forms can be assembled into this single canonical form. Furthermore, the covariant Hamiltonian Noether theory that we construct generalizes Bridges' clever decomposition of water wave conservation laws and is an intrinsic restatement of the constrained variational principles which lead to the existence of water wave instabilities and diagonal periodic pattern formation.

We begin in Section 2 by recalling some of the basic constructions and a few key 
results from the multisymplectic formalism of [8]. In Section 3, we add the structure of connection and intrinsically define our covariant Hamiltonian density. In Section 4 we show that our multisymplectic formalism generalizes the classical theory of particle mechanics, as well Bridges' theory of nonlinear partial differential equations. Section 5 is devoted to our development of a covariant Hamiltonian Noether theory. In Section 6 we show how this theory recovers the classical conservation laws of particle mechanics as well as the new conservation laws proposed by Bridges [2] for studying water waves. Finally, in Section 7 we show how our general theory applies to the study of periodic pattern formation and the instability of waves.

\section{Multisymplectic geometry}

A covariant configuration bundle is a finite-dimensional fibre bundle $\pi_{X Y}: Y \rightarrow X$ over an oriented manifold $X$. In many examples, especially those occurring in relativistic field theories, $X$ is chosen to be spacetime and the fields of interest are sections of this bundle. For nonrelativistic theories, such as nonlinear waves, one typically chooses $X$ to be classical spacetime (i.e. the product of the reals, $\mathbb{R}$, with the spatial variables).

We shall need a little notation. Denote the fibre $\pi_{X Y}^{-1}(x)$ of $Y$ over $x \in X$ by $Y_{x}$ and the tangent space to $X$ at $x$ by $T_{x} X$, etc., and denote sections of $\pi_{X Y}$ by $\Gamma\left(\pi_{X Y}\right)$. We also let $V Y \subset T Y$ be the vertical subbundle; this is the bundle over $Y$ whose fibers are given by

$$
V_{y} Y=\left\{v \in T_{y} Y \mid T \pi_{X Y} \cdot v=0\right\},
$$

where $T \pi_{X Y} \cdot v$ denotes the derivative of the map $\pi_{X Y}$ in the direction $v$.

Just as the covariant configuration bundle is the analogue of the configuration space in particle mechanics, the first jet bundle, defined next, is the field theoretic analogue of the tangent bundle.

Definition $2 \cdot 1$. The first jet bundle $J^{1}(Y)$ is the affine bundle over $Y$ whose fibre over $y \in Y_{x}$ consists of those linear mappings $\gamma: T_{x} X \rightarrow T_{y} Y$ satisfying

$$
T \pi_{X Y} \circ \gamma=\text { identity on } T_{x} X \text {. }
$$

The vector bundle underlying this affine bundle is the bundle whose fibre over $y \in Y_{x}$ is the space $L\left(T_{x} X, V_{y} Y\right)$ of linear mappings of $T_{x} X$ into $V_{y} Y$. Note that for each $\gamma \in J^{1}(Y)_{y}$, we have the splitting

$$
T_{y} Y=\text { image } \gamma \oplus V_{y} Y .
$$

The choice of the first jet bundle $J^{1}(Y)$ is used for the field theoretic tangent bundle for classical field theories whose Lagrangians depend on the point values of the fields and their first derivatives. For higher order field theories one uses higher order jet bundles; see $[\mathbf{8}, \mathbf{9}]$ for references to this literature.

We let $\operatorname{dim} X=n+1$ and the fibre dimension of $Y$ be $N$. Coordinates on $X$ are denoted $x^{\mu}, \mu=1,2, \ldots, n, 0$, and fibre coordinates on $Y$ are denoted by $y^{A}, A=$ $1, \ldots, N$. These induce coordinates $v_{\mu}^{A}$ on the fibres of $J^{1}(Y)$. If $\phi: X \rightarrow Y$ is a section of $\pi_{X Y}$, its tangent map at $x \in X$, denoted $T_{x} \phi$, is an element of $J^{1}(Y)_{\phi(x)}$. Thus, the map $x \mapsto T_{x} \phi$ defines a section of $J^{1}(Y)$ regarded as a bundle over $X$. This section is denoted $j^{1}(\phi)$ and is called the first jet of $\phi$. In coordinates, $j^{1}(\phi)$ is 
given by

$$
x^{\mu} \mapsto\left(x^{\mu}, \phi^{A}\left(x^{\mu}\right), \partial_{\nu} \phi^{A}\left(x^{\mu}\right)\right),
$$

where $\partial_{\nu}=\partial / \partial x^{\nu}$. A section of the bundle $J^{1}(Y) \rightarrow X$ which is the first jet of a section of $Y \rightarrow X$ is said to be holonomic.

The field theoretic analogue of the cotangent bundle is defined next.

Definition $2 \cdot 2$. The dual jet bundle $J^{1}(Y)^{\star}$ is the vector bundle over $Y$ whose fibre at $y \in Y_{x}$ is the set of affine maps from $J^{1}(Y)_{y}$ to $\Lambda^{n+1}(X)_{x}$, the bundle of $(n+1)$-forms on $X$.

A smooth section of $J^{1}(Y)^{\star}$ is therefore an affine bundle map of $J^{1}(Y)$ to $\Lambda^{n+1}(X)$ covering $\pi_{X Y}$. We choose affine maps since $J^{1}(Y)$ is an affine bundle and we map into $\Lambda^{n+1}(X)$ since we are ultimately thinking of integration as providing the pairing on sections.

Fibre coordinates on $J^{1}(Y)^{\star}$ are $\left(p, p_{A}{ }^{\mu}\right)$, which correspond to the affine map given in coordinates by

$$
v_{\mu}^{A} \mapsto\left(p+p_{A}^{\mu} v_{\mu}^{A}\right) d^{n+1} x,
$$

where $d^{n+1} x=d x^{1} \wedge \cdots \wedge d x^{n} \wedge d x^{0}$.

Analogous to the canonical one- and two-forms on a cotangent bundle, there are canonical forms on $J^{1}(Y)^{\star}$. To define these another description of $J^{1}(Y)^{\star}$ will be convenient. Namely, let $\Lambda:=\Lambda^{n+1}(Y)$ denote the bundle of $(n+1)$-forms on $Y$, with fibre over $y \in Y$ denoted by $\Lambda_{y}$ and with projection $\pi_{Y \Lambda}: \Lambda \rightarrow Y$. Let $Z \subset \Lambda$ be the subbundle whose fibre is given by

$$
\left.\left.Z_{y}=\left\{z \in \Lambda_{y} \mid v\right\lrcorner(w\lrcorner z\right)=0 \text { for all } v, w \in V_{y} Y\right\},
$$

where $v\lrcorner$ denotes left interior multiplication by $v$.

Elements of $Z$ can be be written uniquely as

$$
z=p d^{n+1} x+p_{A}^{\mu} d y^{A} \wedge d^{n} x_{\mu},
$$

where

$$
\left.d^{n} x_{\mu}=\partial_{\mu}\right\lrcorner d^{n+1} x \quad \text { and, as before, } \quad \partial_{\mu}=\frac{\partial}{\partial x^{\mu}} .
$$

Hence, fibre coordinates for $Z$ are also $\left(p, p_{A}{ }^{\mu}\right)$.

Corresponding to equating the coordinates $\left(x^{\mu}, y^{A}, p, p_{A}^{\mu}\right)$ of $Z$ and of $J^{1}(Y)^{\star}$, there is a vector bundle isomorphism

$$
\Phi: Z \rightarrow J^{1}(Y)^{\star}
$$

Intrinsically, $\Phi$ is defined by pull-back:

$$
\Phi(z)(\gamma)=\gamma^{*} z \in \Lambda^{n+1}(X)_{x},
$$

where $z \in Z_{y}, \gamma \in J^{1}(Y)_{y}$ and $x=\pi_{X Y}(y)$. Using fibre coordinates $v^{A}{ }_{\mu}$ for $\gamma$, the preceding equation becomes

$$
\gamma^{*} d x^{\mu}=d x^{\mu} \quad \text { and } \quad \gamma^{*} d y^{A}=v_{\mu}^{A} d x^{\mu}
$$

and so

$$
\gamma^{*}\left(p d^{n+1} x+p_{A}^{\mu} d y^{A} \wedge d^{n} x_{\mu}\right)=\left(p+p_{A}^{\mu} v_{\mu}^{A}\right) d^{n+1} x,
$$

where we have used $d x^{\nu} \wedge d^{n} x_{\mu}=\delta_{\mu}^{\nu} d^{n+1} x$. 
One shows that the inverse of $\Phi$ can also be defined intrinsically, although it is somewhat more complicated, and thus the spaces $J^{1}(Y)^{\star}$ and $Z$ are canonically isomorphic as vector bundles over $Y$.

There are canonical forms on $Z$ and the isomorphism between $J^{1}(Y)^{\star}$ and $Z$ can be used to transfer these to $J^{1}(Y)^{\star}$. We first define the canonical $(n+1)$-form $\Theta_{\Lambda}$ on $\Lambda$ by

$$
\begin{aligned}
\Theta_{\Lambda}(z)\left(u_{1}, \ldots, u_{n+1}\right) & =z\left(T \pi_{Y \Lambda} \cdot u_{1}, \ldots, T \pi_{Y \Lambda} \cdot u_{n+1}\right) \\
& =\left(\pi_{Y \Lambda}^{*} z\right)\left(u_{1}, \ldots, u_{n+1}\right)
\end{aligned}
$$

where $z \in \Lambda$ and $u_{1}, \ldots, u_{n+1} \in T_{z} \Lambda$. Define the canonical $(n+2)$-form $\Omega_{\Lambda}$ on $\Lambda$ by

$$
\Omega_{\Lambda}=-d \Theta_{\Lambda} .
$$

Note that if $n=0$ (i.e. $X$ is one-dimensional), then $\Lambda=T^{*} Y$ and $\Theta_{\Lambda}$ is the standard canonical one-form. If $i_{\Lambda Z}: Z \rightarrow \Lambda$ denotes the inclusion, the canonical $(n+1)$-form $\Theta$ on $Z$ is defined by

$$
\Theta=i_{\Lambda Z}^{*} \Theta_{\Lambda}
$$

and the canonical $(n+2)$-form $\Omega$ on $Z$ is defined by

$$
\Omega=-d \Theta=i_{\Lambda Z}^{*} \Omega_{\Lambda} .
$$

The pair $(Z, \Omega)$ is called multiphase space or covariant phase space. It is an example of a multisymplectic manifold.

Using $(2 \cdot 7),(2 \cdot 12),(2 \cdot 13),(2 \cdot 14)$ and $(2 \cdot 15)$, one finds that the coordinate expression for $\Theta$ is

$$
\Theta=p_{A}{ }^{\mu} d y^{A} \wedge d^{n} x_{\mu}+p d^{n+1} x,
$$

and so

$$
\Omega=d y^{A} \wedge d p_{A}^{\mu} \wedge d^{n} x_{\mu}-d p \wedge d^{n+1} x .
$$

Let the Lagrangian density $\mathscr{L}: J^{1}(Y) \rightarrow \Lambda^{n+1}(X)$, be a given smooth bundle map over $X$. In coordinates, we write

$$
\mathscr{L}(\gamma)=L\left(x^{\mu}, y^{A}, v_{\mu}^{A}\right) d^{n+1} x .
$$

The corresponding covariant Legendre transformation associated with $\mathscr{L}$ is a fibre preserving map over $Y, \mathbb{F} \mathscr{L}: J^{1}(Y) \rightarrow J^{1}(Y)^{\star} \cong Z$, whose intrinsic definition follows.

Definition 2.3. If $\gamma \in J^{1}(Y)_{y}$, we define $\mathbb{F} \mathscr{L}(\gamma) \in J^{1}(Y)_{y}^{\star}$ (where $y \in Y_{x}$ ) to be the first order vertical Taylor approximation to $\mathscr{L}$ :

$$
\mathbb{F} \mathscr{L}(\gamma) \cdot \gamma^{\prime}=\mathscr{L}(\gamma)+\left.\frac{d}{d \varepsilon}\right|_{\varepsilon=0} \mathscr{L}\left(\gamma+\varepsilon\left(\gamma^{\prime}-\gamma\right)\right)
$$

where $\gamma^{\prime} \in J^{1}(Y)_{y}$.

A straightforward calculation shows that the covariant Legendre transformation is given in coordinates by

$$
p_{A}{ }^{\mu}=\frac{\partial L}{\partial v_{\mu}^{A}}, \text { and } \quad p=L-\frac{\partial L}{\partial v^{A}{ }_{\mu}} v_{\mu}^{A} .
$$

Notice that formally, the second of these equations defines the (negative of the) 
energy while the first one is reminiscent of the usual relation $p_{i}=\partial L / \partial \dot{q}^{i}$ from classical mechanics. One of the nice features of the covariant Legendre transformation is how these two basic aspects of the Legendre transformation arise from a single construction.

Definition 2.4. The Cartan form is the $(n+1)$-form $\Theta_{\mathscr{L}}$ on $J^{1}(Y)$ defined by

$$
\Theta_{\mathscr{L}}=(\mathbb{F} \mathscr{L})^{*} \Theta
$$

where $\Theta$ is the canonical $(n+1)$-form on $Z$. We also define the $(n+2)$-form $\Omega_{\mathscr{L}}$ by

$$
\Omega_{\mathscr{L}}=-d \Theta_{\mathscr{L}}=(\mathbb{F} \mathscr{L})^{*} \Omega
$$

where $\Omega=-d \Theta$ is the canonical $(n+2)$-form on $Z$.

\section{The covariant Hamiltonian}

In this section we develop an intrinsic covariant (or multisymplectic) Hamiltonian formalism. We begin by noting that the covariant Legendre transformation $\mathbb{F} \mathscr{L}: J^{1}(Y) \rightarrow J^{1}(Y)^{\star}$ is never a fibre bundle diffeomorphism since $\operatorname{dim} J^{1}(Y)^{\star}=$ $\operatorname{dim} J^{1}(Y)+1$; nevertheless, it is appropriate in many examples to require $\mathbb{F} \mathscr{L}$ to be a smooth bundle diffeomorphism over $Y$ onto its image. In fact, from the second equation in $(2 \cdot 20)$, the image of $\mathbb{F} \mathscr{L}$ defines the primary constraint of the theory.

Definition $3 \cdot 1$. We say that $\mathscr{L}$ is regular if the image of the first jet bundle under the covariant Legendre transformation $P_{\mathscr{L}}:=\mathbb{F} \mathscr{L}\left(J^{1}(Y)\right)$ is a smooth manifold and if $\mathbb{F} \mathscr{L}$ is a diffeomorphism onto $P_{\mathscr{L}}$. We call $P_{\mathscr{L}}$ the primary constraint manifold.

One should note that many field theories, such as the vacuum Maxwell equations and many others, especially relativistic ones, are not regular because of the presence of constraints (such as div $\mathbf{E}=0$ for Maxwell's equations). We are assuming regularity only for simplicity and because it is appropriate for the examples we have in mind. Gotay et al. [8] deal with the more general case in a Lagrangian formalism.

At this point we introduce the additional structure of a connection. While connections are not particularly needed for the Lagrangian side of field theory, they seem to be essential for the development of an intrinsic Hamiltonian formalism. We recall the definition of an (Eheresmann) connection as a vertical-valued one-form.

Definition 3.2. A connection on $Y$ is a vector bundle map $\mathfrak{A}: T Y \rightarrow V Y$ such that on each fibre over $y \in Y, \mathfrak{A}: T_{y} Y \rightarrow V_{y} Y$ satisfies

$$
\mathfrak{A}=\text { identity on } V Y \text {. }
$$

The horizontal space at each point $y \in Y$ is defined by $\operatorname{hor}_{y}=\operatorname{ker} \mathfrak{A}_{y}$, so that we have $T_{y} Y=\operatorname{hor}_{y} \oplus V_{y} Y$.

In coordinates, the action of $\mathfrak{A}$ on a tangent vector to $Y$, namely $\left(v^{\nu}, v^{A}\right)$ is written as $\left(0, v^{A}+\mathfrak{A}_{\mu}^{A} v^{\mu}\right)$. This defines the coordinate expression for the connection. We remark that it is not entirely necessary to a priori explicitly introduce a connection if one wishes to define the Hamiltonian locally in a coordinate chart, and then use coordinate patches to obtain a global characterization; however, the process of producing a coordinate independent global definition is tantamount to producing a connection. 
Next, we reexpress the covariant Legendre transformation $\mathbb{F} \mathscr{L}$ in terms of a vertical derivative of functions on $J^{1}(Y)$.

Definition 3.3. For any $x \in X$ and $y \in Y_{x}$, let $U \subset J^{1}(Y)_{y}$ be an open subset and let $\mathscr{S} \in C^{1}\left(U, \Lambda^{n+1}(X)_{x}\right)$. Then the covariant derivative of $S$ associated with the connection $\mathfrak{A}$ maps $U$ into $C^{0}\left(J^{1}(Y)_{y}, \Lambda^{n+1}(X)_{x}\right)$ and is defined, for any $\gamma \in U$ and $\gamma^{\prime} \in J^{1}(Y)_{y}$, by

$$
D_{\mathfrak{Y}} \mathscr{S}(\gamma) \cdot \gamma^{\prime}=\left.\frac{d}{d \varepsilon}\right|_{\varepsilon=0} \mathscr{S}\left(\gamma+\varepsilon \mathfrak{A}\left(\gamma^{\prime}\right)\right) .
$$

It is then natural to consider the covariant derivative of the smooth bundle map $\mathscr{L}: \pi_{X, J^{1}(Y)} \rightarrow \pi_{X, \Lambda^{n+1}(X)}$, so that, using (3·2), the Legendre transformation can be written as

$$
\mathbb{F} \mathscr{L}(\gamma) \cdot \gamma^{\prime}=\left[\mathscr{L}(\gamma)-D_{\mathfrak{Q}} \mathscr{L}(\gamma) \cdot \gamma\right]+D_{\mathfrak{Q}} \mathscr{L}(\gamma) \cdot \gamma^{\prime}
$$

for all $\gamma, \gamma^{\prime} \in J^{1}(Y)_{y}$ and $y \in Y_{x}$. Note that this expression is affine with the first two terms being the constant terms and the last one being the linear term.

We may now define the covariant Hamiltonian density on the primary constraint manifold $P_{\mathscr{L}}$.

Definition 3.4. For a regular Lagrangian, the corresponding covariant Hamiltonian $\mathscr{H}: P_{\mathscr{L}} \rightarrow \Lambda^{n+1}(X)$ is defined by

$$
\mathscr{H}(z)=D_{\mathfrak{Q}} \mathscr{L}(\gamma) \cdot \gamma-\mathscr{L}(\gamma),
$$

where $z=\mathbb{F} \mathscr{L}(\gamma)$.

In coordinates, we may write $\mathscr{H}=H d^{n+1} x$ where

$$
H=\frac{\partial L}{\partial v_{\mu}^{A}}\left(v_{\mu}^{A}+\mathfrak{A}_{\mu}^{A}\right)-L .
$$

Notice that the covariant Hamiltonian is well defined under the assumption of regularity; namely, the map $\gamma \mapsto z=\mathbb{F} \mathscr{L}(\gamma)$ from $J^{1}(Y)$ to $P_{\mathscr{L}}$ is a diffeomorphism.

We coordinatize the primary constraint manifold by $\left(x^{\mu}, y^{A}, p_{A}^{\mu}\right)$ with $p$ now expressed in terms of the other variables by rewriting the preceding expression for $H$ as

$$
H=\frac{\partial L}{\partial v_{\mu}^{A}} \mathfrak{I}_{\mu}^{A}-p,
$$

regarded as an implicit equation for $p$.

Let $i_{J^{1}(Y)^{\star}, P_{\mathscr{L}}}: P_{\mathscr{L}} \rightarrow J^{1}(Y)^{\star}$ denote the inclusion. We may pull-back the canonical $(n+1)$ - and $(n+2)$-forms on $J^{1}(Y)^{\star}$ to $P_{\mathscr{L}}$ and obtain (using a notation to remind us that this takes the Hamiltonian point of view):

$$
\left.\begin{array}{l}
\Theta_{\mathscr{H}}=i_{J^{1}(Y)^{\star}, P_{\mathscr{P}}}^{*} \Theta, \\
\Omega_{\mathscr{H}}=i_{J^{1}(Y)^{\star}, P_{\mathscr{P}}}^{*} \Omega .
\end{array}\right\}
$$

In canonical coordinates, we have

$$
\left.\begin{array}{c}
\Theta_{\mathscr{H}}=p_{A}{ }^{\mu} d y^{A} \wedge d^{n} x_{\mu}+\left(p_{A}{ }^{\mu} \mathfrak{O}_{\mu}^{A}-H\right) \wedge d^{n+1} x \\
\Omega_{\mathscr{H}}=d y^{A} \wedge d p_{A}^{\mu} \wedge d^{n} x_{\mu}+\left[\frac{\partial H}{\partial y^{A}} d y^{A}+\left(\frac{\partial H}{\partial p_{A}^{\mu}}-\mathfrak{Q}_{\mu}^{A}\right) d p_{A}^{\mu}\right] \wedge d^{n+1} x
\end{array}\right\}
$$


For many important examples, we will consider $X$ as the classical spacetime manifold with the locally trivial connection which is simply the natural projection whose action in coordinates is $\left(0, v^{A}\right)$, i.e. the components $\mathfrak{I}_{\mu}^{A}=0$.

Definition 3.5. Let $\phi \in \Gamma\left(\pi_{X Y}\right)$, and $j^{1}(\phi)$ its first jet. A section $z$ of $\pi_{X, P_{\varphi}}$ is called conjugate to $j^{1}(\phi)$ if $z=\mathbb{F} \mathscr{L} \circ j^{1}(\phi)$. In this case, we shall write $\tilde{j}^{1}(\phi)$ for $z$ and say that $z$ is holonomic.

Definition 3.6. A holonomic section $z$ of $P_{\mathscr{L}}$ is called Hamiltonian for $\mathscr{H}$ if

$$
\left.z^{*}(U\lrcorner \Omega_{\mathscr{H}}\right)=0 .
$$

for any $U \in T\left(P_{\mathscr{L}}\right)$. We also refer to the system of equations (3.7) regarded as differential equations for $z$ as the multihamiltonian system of equations associated to $\mathscr{H}$.

Lemma 3.1. If $\mathbb{F} \mathscr{L}: J^{1}(Y) \rightarrow P_{\mathscr{L}}$ is a fibre bundle diffeomorphism over $Y$ and if $\phi$ is in $\Gamma\left(\pi_{X Y}\right)$, then the following are equivalent:

(i) $\left.\tilde{j}^{1}(\phi)^{*}(U\lrcorner \Omega_{\mathscr{H}}\right)=0$ for any $U \in T\left(P_{\mathscr{L}}\right)$;

(ii) $\left.j^{1}(\phi)^{*}(W\lrcorner \Omega_{\mathscr{L}}\right)=0$ for any $W \in T\left(J^{1}(Y)\right)$.

Proof. Assume (i) holds and let $U \in T\left(P_{\mathscr{L}}\right)$. Since $\mathbb{F} \mathscr{L}$ is a fibre bundle diffeomorphism, there exists $W \in T\left(J^{1}(Y)\right)$ such that $T \mathbb{F} \mathscr{L} \circ W=U \circ \mathbb{F} \mathscr{L}$. Hence,

$$
\begin{aligned}
\left.0=\tilde{j}^{1}(\phi)^{*}(U\lrcorner \Omega_{\mathscr{H}}\right) & \left.=j^{1}(\phi)^{*} \mathbb{F} \mathscr{L}^{*}(T \mathbb{F} \mathscr{L} \cdot W\lrcorner \Omega_{\mathscr{H}}\right) \\
& \left.=j^{1}(\phi)^{*}\left(\mathbb{F} \mathscr{L}^{*} T \mathbb{F} \mathscr{L} \cdot W\right\lrcorner \mathbb{F} \mathscr{L}^{*} \Omega_{\mathscr{H}}\right) \\
& \left.=j^{1}(\phi)^{*}(W\lrcorner \Omega_{\mathscr{L}}\right) .
\end{aligned}
$$

Using the same argument, the inverse function theorem guarantees that the converse holds as well.

We are thus led to the following conclusion.

Theorem 3.1. If $\mathbb{F} \mathscr{L}: J^{1}(Y) \rightarrow P_{\mathscr{L}}$ is a fibre bundle diffeomorphism over $Y$ and $\phi \in \Gamma\left(\pi_{X Y}\right)$, then the following are equivalent:

(i) $\phi$ is a stationary point of $\int_{X} \mathscr{L}\left(j^{1}(\phi)\right)$;

(ii) $\tilde{j}^{1}(\phi)$ is a Hamiltonian section for $\mathscr{H}$.

Before we prove the theorem, we state the following definition and lemma.

Definition 3.7. A (finite) variation of $\phi$ is a curve $\phi_{\lambda}=\eta_{\lambda} \circ \phi$, where $\eta_{\lambda}$ is the flow of a vertical vector field $V$ on $Y$ which is compactly supported in $X$. One says that $\phi$ is a stationary point of the action if

$$
\left.\frac{d}{d \lambda}\left[\int_{X} \mathscr{L}\left(j^{1}\left(\phi_{\lambda}\right)\right)\right]\right|_{\lambda=0}=0
$$

for all variations $\phi_{\lambda}$ of $\phi$.

Lemma 3·2. If $\mathbb{F} \mathscr{L}: J^{1}(Y) \rightarrow P_{\mathscr{L}}$ is a fibre bundle diffeomorphism, then

$$
\left.\tilde{j}^{1}(\phi)^{*}(U\lrcorner \Omega_{\mathscr{H}}\right)=0
$$


for any $U$ which is $\pi_{Y, P_{\mathscr{P}}}$-vertical or is tangent to $\tilde{j}^{1}(\phi)$. Similarly,

$$
\left.j^{1}(\phi)^{*}(W\lrcorner \Omega_{\mathscr{L}}\right)=0
$$

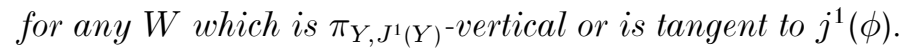

Proof. Since $\mathbb{F} \mathscr{L}$ is a fibre-preserving bundle diffeomorphism, for any $\pi_{Y, P_{\mathscr{L}}}$-vertical $U$, there exists a $\pi_{Y, J^{1}(Y)}$-vertical $W$ such that $T \mathbb{F} \mathscr{L} \circ W=U \circ \mathbb{F} \mathscr{L}$. Using canonical coordinates, let us write $U$ and $W$ as

$$
U=U_{A}{ }^{\mu} \frac{\partial}{\partial p_{A}{ }^{\mu}}, \quad \text { and } \quad W=W_{\mu}{ }^{A} \frac{\partial}{\partial v^{A}{ }_{\mu}} .
$$

A calculation using $(3 \cdot 6)$ shows that

$$
U\lrcorner \Omega_{\mathscr{H}}=U_{A}^{\mu}\left(d y^{A} \wedge d^{n} x_{\mu}+\left(\frac{\partial H}{\partial p_{A} \mu}-\mathfrak{A}_{\mu}^{A}\right) d^{n+1} x\right) .
$$

Hence, using Lemma $3 \cdot 1$, we have that

$$
\left.\tilde{j}^{1}(\phi)^{*}(U\lrcorner \Omega_{\mathscr{C}}\right)=j^{1}(\phi)^{*}\left(-W_{v_{\nu}^{B}} \frac{\partial L}{\partial v_{\mu}^{A} \partial v_{\nu}^{B}}\left(d y^{A} \wedge d^{n} x_{\mu}-v_{\mu}^{A} d^{n+1} x\right)\right),
$$

which vanishes using $(2 \cdot 10)$. On the other hand, if $U$ is tangent to the graph of $\tilde{j}^{1}(\phi)$, then $U=T \tilde{j}^{1}(\phi) . v$ for some $v \in T X$ so that

$$
\left.\left.\left.\tilde{j}^{1}(\phi)^{*}(U\lrcorner \Omega_{\mathscr{C}}\right)=\tilde{j}^{1}(\phi)^{*}\left(\left(T \tilde{j}^{1}(\phi) \cdot v\right)\right\lrcorner \Omega_{\mathscr{H}}\right)=v\right\lrcorner\left(\tilde{j}^{1}(\phi)^{*} \Omega_{\mathscr{C}}\right),
$$

which vanishes since $\tilde{j}^{1}(\phi)^{*} \Omega_{\mathscr{L}}$ is an $(n+2)$-form on the $(n+1)$-manifold $X$. The identical argument works for $W$.

The proof of Lemma $3 \cdot 2$ shows that in canonical coordinates

$$
\frac{\partial H}{\partial p_{A}{ }^{\mu}}=\nu_{\mu}^{A}+\mathfrak{A}_{\mu}^{A},
$$

and in the case that $U=U_{y^{A}} \partial / \partial y^{A}$ and that $\left.\tilde{j}^{1}(\phi)^{*}(U\lrcorner \Omega_{\mathscr{H}}\right)=0$, we obtain that

$$
\frac{\partial H}{\partial y^{A}}=-\frac{\partial p_{A}^{\mu}}{\partial x^{\mu}} .
$$

Thus, $(3 \cdot 10)$ and $(3 \cdot 11)$ are the coordinate expressions for a multihamiltonian system.

Proof of Theorem 3.1 Let $\phi_{\lambda}=\eta_{\lambda} \circ \phi$ of $\phi$ be a variation corresponding to a $\pi_{X Y}$ vertical vector field $V$ on $Y$ with compact support in $X$. Using $(2 \cdot 7)$ we find that $\mathscr{L}\left(j^{1}(\phi)\right)=j^{1}(\phi)^{*} \Theta_{\mathscr{L}}$ and hence

$$
\begin{aligned}
\left.\frac{d}{d \lambda}\left[\int_{X} \mathscr{L}\left(j^{1}\left(\phi_{\lambda}\right)\right)\right]\right|_{\lambda=0} & =\left.\frac{d}{d \lambda} \int_{X} j^{1}\left(\phi_{\lambda}\right)^{*} \Theta_{\mathscr{L}}\right|_{\lambda=0} \\
& \left.=\frac{d}{d \lambda}\left[\int_{X} j^{1}(\phi)^{*} j^{1}\left(\eta_{\lambda}\right)^{*} \Theta_{\mathscr{L}}\right)\right]\left.\right|_{\lambda=0} \\
& =\int_{X} j^{1}(\phi)^{*} \mathfrak{S}_{j^{1}(V)} \Theta_{\mathscr{L}}
\end{aligned}
$$

where

$$
j^{1}(V)=\left.\frac{d}{d \lambda} j^{1}\left(\eta_{\lambda}\right)\right|_{\lambda=0}
$$


is the jet prolongation of $V$ to $J^{1}(Y)$ (see Definition 5·4, if necessary). Using Cartan's magic formula, we get $\left.\left.\mathfrak{L}_{W} \Theta_{\mathscr{L}}=-W\right\lrcorner \Omega_{\mathscr{L}}+d(W\lrcorner \Theta_{\mathscr{L}}\right)$, which, together with (3.12), gives

$$
\left.\begin{array}{rl}
\left.\frac{d}{d \lambda}\left[\int_{X} \mathscr{L}\left(j^{1}\left(\phi_{\lambda}\right)\right)\right]\right|_{\lambda=0}= & \left.-\int_{X} j^{1}(\phi)^{*}\left(j^{1}(V)\right\lrcorner \Omega_{\mathscr{L}}\right) \\
& \left.+\int_{X} d j^{1}(\phi)^{*}\left(j^{1}(V)\right\lrcorner \Theta_{\mathscr{L}}\right) \\
= & \left.-\int_{X} j^{1}(\phi)^{*}\left(j^{1}(V)\right\lrcorner \Omega_{\mathscr{L}}\right)
\end{array}\right\}
$$

by Stokes' theorem and the fact that $V$, and hence $j^{1}(V)$, is compactly supported in $X$. Lemma $3 \cdot 1$ together with $(3 \cdot 13)$ shows that (ii) implies (i).

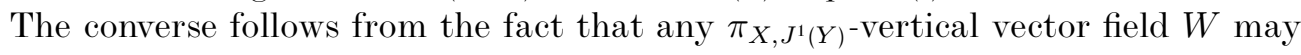
be decomposed as

$$
W=j^{1}(V)+W_{1},
$$

where $V$ is $\pi_{X Y^{-v}}$-vertical and $W_{1}$ is $\pi_{Y, J^{1}(Y)}$-vertical. Then, if (i) holds, Lemma $3 \cdot 1$ and Lemma $3 \cdot 2$ together with $(3 \cdot 13)$ show that

$$
\left.\int_{X} \tilde{j}^{1}(\phi)^{*}(U\lrcorner \Omega_{\mathscr{H}}\right)=0
$$

for all vector fields $U$ on $P_{\mathscr{L}}$ with compact support in $X$. Since the space of smooth vector fields on $J^{1}(Y)$ is a module over the ring of smooth functions on $X$, an argument like that in the fundamental lemma of the calculus of variations shows that the integrand must vanish for all vector fields $U \in T\left(P_{\mathscr{L}}\right)$ with compact support in $X$. A partition of unity argument then shows that (ii) implies (i).

In the next section, we shall demonstrate the machinery of our intrinsic development on two examples: classical mechanics and nonlinear partial differential equations. We note that the essence of both of the following examples are $(3 \cdot 10)$ and $(3 \cdot 11)$. For a development of a generalized Hamiltonian structure based on Hamiltonian vector fields that seems well-suited for ordinary differential equations, we refer the reader to $[6]$.

\section{Particle mechanics and nonlinear partial differential equations}

In this section, we show that our multisymplectic Hamiltonian formalism generalizes classical particle mechanics and is a natural setting for nonlinear Hamiltonian partial differential equations.

\subsection{Particle mechanics}

For non-relativistic classical mechanics with a configuration manifold $Q$ (of dimension $N$ ), we choose $X=\mathbb{R}$ (so that $n=0$ ) and $Y=\mathbb{R} \times Q$. In this case, $J^{1}(Y)=\mathbb{R} \times T Q$, and the cross-product induces a (flat) connection $\mathfrak{A}: \mathbb{R} \times T Q \rightarrow T Q$. The dual jet bundle is given by $J^{1}(Y)^{\star}=T^{*} \mathbb{R} \times T^{*} Q$ and has canonical coordinates $\left(t, p, q^{1}, \ldots, q^{N}, p_{1}, \ldots, p_{N}\right)$.

Given a Lagrangian in the usual sense $L: \mathbb{R} \times T Q \rightarrow \mathbb{R}$, we define $\mathscr{L}: J^{1}(Y) \rightarrow \Lambda^{1}(Y)$ by

$$
\mathscr{L}\left(t, q^{A}, \dot{q}^{A}\right)=L\left(t, q^{A}, \dot{q}^{A}\right) d t .
$$


The covariant Legendre transformation is the map $\mathbb{F} \mathscr{L}: J^{1}(Y) \rightarrow J^{1}(Y)^{*}$, i.e. $\mathbb{F} \mathscr{L}: \mathbb{R} \times T Q \rightarrow T^{*} \mathbb{R} \times T^{*} Q$ given by

$$
\mathbb{F} \mathscr{L}\left(t, q^{i}, \dot{q}^{i}\right)=\left(t, L\left(t, q^{A}, \dot{q}^{A}\right)-p_{A} \dot{q}^{A}, q^{A}, p_{A}\right),
$$

where $p_{A}=\partial L / \partial \dot{q}^{A}$. In this case, it is clear that $P_{\mathscr{L}}=\mathbb{R} \times T^{*} Q$ (as a subbundle of $\left.T^{*} \mathbb{R} \times T^{*} Q\right)$ with coordinates $\left(t, q^{A}, p_{A}\right)$. Assume that the Legendre transformation is nondegenerate in the usual sense so that $\mathbb{F} \mathscr{L}: \mathbb{R} \times T Q \rightarrow P_{\mathscr{L}}$ is a vector bundle diffeomorphism over $\mathbb{R}$ and the corresponding Hamiltonian $H: \mathbb{R} \times T^{*} Q \rightarrow \mathbb{R}$ is well defined. The function $H$ corresponds to the density $\mathscr{H}: P_{\mathscr{L}} \rightarrow \Lambda^{1}(X)$ as

$$
\mathscr{H}\left(t, q^{A}, p_{A}\right)=H\left(t, q^{A}, p_{A}\right) d t,
$$

where

$$
\mathscr{H}(z)=D_{\mathfrak{H}} \mathscr{L}(\gamma) \cdot \gamma-\mathscr{L}(\gamma), \quad z=\mathbb{F} \mathscr{L}(\gamma) \in P_{\mathscr{L}}
$$

has the coordinate expression

$$
\mathscr{H}\left(t, q^{A}, p_{A}\right)=\left(p_{A} q^{A}-L\left(t, q^{A}, \dot{q}^{A}\right)\right) d t .
$$

In this case, we obtain the usual symplectic 2 -form on extended phase space

$$
\Omega_{\mathscr{H}}=d q^{A} \wedge d p_{A}+d H \wedge d t .
$$

Proposition 4.1. Let $\bar{V}$ be a vector field on $P_{\mathscr{L}}$ with integral curve $\tilde{j}^{1}(\phi)$. Then $\bar{V}$ is a Hamiltonian vector field for $H$ if and only if $\tilde{j}^{1}(\phi)$ is a Hamiltonian section for $\mathscr{H}$.

Proof. In coordinates, let $\bar{v}=\left(1, V_{q}^{A}, V_{p}^{B}\right)$ and let $\left(U_{t}, U\right) \equiv\left(U_{t}, U_{q}^{A}, U_{p}^{B}\right)$ be the coordinates for an arbitrary vector field $\bar{U} \in T\left(P_{\mathscr{L}}\right)$. Then

$$
i_{\bar{U}} \Omega_{\mathscr{H}}=U_{p}^{B} d q^{A}-U_{q}^{A} d p^{B}+(d H . U) d t-U_{t} d H,
$$

and the pull-back of $(4 \cdot 1)$ under $\tilde{j}^{1}(\phi)$ vanishes if and only if

$$
\dot{q}^{A}=-\frac{\partial H}{\partial p_{A}}, \quad \dot{p}_{B}=\frac{\partial H}{\partial q^{B}},
$$

and

$$
d H . U=0 .
$$

\subsection{Nonlinear partial differential equations}

To motivate the exposition, consider the nonlinear wave equation given by

$$
\frac{\partial^{2} \phi}{\partial x^{0^{2}}}-\triangle \phi-V^{\prime}(\phi)=0, \quad \phi \in \Gamma\left(\pi_{X Y}\right),
$$

where $\triangle$ is the Laplace-Beltrami operator and $V$ is a real-valued $C^{\infty}$ function of one variable.

We will show that $(3 \cdot 7)$ along the holonomic section $\tilde{j}^{1}(\phi)$ is equivalent to the nonlinear partial differential equation (4.4) as well as the Bridges [4] coordinate representation. For clarity of presentation, we will consider only one spatial dimension. In terms of our general notation, we set $X=\mathbb{R}^{2}(n=1)$ and $Y=\mathbb{R}^{2} \times M$, so that sections of $Y$ have the coordinate expressions $\left(x^{0}, x^{1}, \phi\right)$, and $T Y=\mathbb{R}^{2} \times T M$. The cross-product once again induces a flat connection defined by the natural projection $\mathfrak{U}: \mathbb{R}^{2} \times T M \rightarrow T M$. 
The first jet bundle $J^{1}(Y)$ is a five-dimensional manifold and sections of $J^{1}(Y)$ have local coordinates $\left(x^{0}, x^{1}, \phi, \partial \phi / \partial x^{0}, \partial \phi / \partial x^{1}\right)$. The affine dual $J^{1}(Y)^{\star}$ is six-dimensional with its sections having the local coordinates $\left(x^{0}, x^{1}, \phi, p, p^{0}, p^{1}\right)$. The Lagrangian density $\mathscr{L}: J^{1}(Y) \rightarrow \Lambda^{2}(X)$ is expressed as

$$
\mathscr{L}\left(x^{0}, x^{1}, \phi, \frac{\partial \phi}{\partial x^{0}}, \frac{\partial \phi}{\partial x^{1}}\right)=L\left(x^{0}, x^{1}, \phi \frac{\partial \phi}{\partial x^{0}}, \frac{\partial \phi}{\partial x^{1}}\right) d x^{1} \wedge d x^{0}
$$

which, for the case of the nonlinear wave equation is

$$
\mathscr{L}\left(x^{0}, x^{1}, \phi, \frac{\partial \phi}{\partial x^{0}}, \frac{\partial \phi}{\partial x^{1}}\right)=\left[\frac{1}{2}\left(\left\{\partial_{0} \phi\right\}^{2}-\left\{\partial_{1} \phi\right\}^{2}\right)+V(\phi)\right] d x^{1} \wedge d x^{0} .
$$

In this setting, the covariant Legendre transformation $\mathbb{F} \mathscr{L}: J^{1}(Y) \rightarrow J^{1}(Y)^{\star}$ is given by

$$
\begin{aligned}
\mathbb{F} \mathscr{L} & \left(x^{0}, x^{1}, \phi, \frac{\partial \phi}{\partial x^{0}}, \frac{\partial \phi}{\partial x^{1}}\right) \\
& =\left[x^{0}, x^{1}, p^{0} \frac{\partial \phi}{\partial x^{0}}+p^{1} \frac{\partial \phi}{\partial x^{1}} L\left(x^{0}, x^{1}, \phi, \frac{\partial \phi}{\partial x^{0}}, \frac{\partial \phi}{\partial x^{1}}\right), \phi, \frac{\partial \phi}{\partial x^{0}}, \frac{\partial \phi}{\partial x^{1}}\right],
\end{aligned}
$$

where $p^{\mu}=\partial L / \partial \phi_{\mu}$ and $\phi_{\mu}=\partial \phi / \partial x^{\mu}$. When $L$ is regular (it is for the nonlinear wave equation), we have the primary constraint subbundle $P_{\mathscr{L}}:=\pi_{\mathbb{R}^{2}, \mathbb{R}^{2} \times \mathbb{R}^{3}} \subset J^{1}(Y)^{\star}$ with coordinates $\left(x^{0}, x^{1}, \phi, p^{0}, p^{1}\right)$, and the Hamiltonian density on $P_{\mathscr{L}}$ is written in coordinates as

$$
\mathscr{H}\left(x^{0}, x^{1}, \phi, p, p^{0}, p^{1}\right)=\left[p^{0} \frac{\partial \phi}{\partial x^{0}}+p^{1} \frac{\partial \phi}{\partial x^{1}}-L\left(x^{0}, x^{1}, \phi, \frac{\partial \phi}{\partial x^{0}}, \frac{\partial \phi}{\partial x^{1}}\right)\right] d x^{1} \wedge x^{0},
$$

while the canonical 3 -form on $J^{1}(Y)^{\star}$ is given by

$$
\Omega_{\mathscr{H}}=-d \phi \wedge d p^{0} \wedge d x^{1}+d \phi \wedge d p^{1} \wedge d x^{0}+d H \wedge d x^{1} \wedge d x^{0} .
$$

We note that in this case, by global triviality, we may identify $P_{\mathscr{L}}$ with $\pi_{\mathbb{R}^{2}, \mathbb{R}^{3}}$.

Bridges [4] considers this scalar field theory with the manifold $M=\mathbb{R}$ and a Lagrangian $L$ that has no explicit dependence on time or space. (In particular, all of the fibres of both $J^{1}(Y)$ and $P_{\mathscr{L}}$ are identical over $X$ and identified with $\mathbb{R}^{3}$.) $\mathrm{He}$ obtains the following partial differential equation for $\mathscr{Z} \equiv\left(\phi, p^{0}, p^{1}\right)$ :

$$
M \frac{\partial \mathscr{Z}}{\partial x^{0}}+K \frac{\partial \mathscr{Z}}{\partial x^{1}}=-d H(\mathscr{Z}),
$$

where the $3 \times 3$ matrices $M$ and $K$ are defined to be

$$
M=\left[\begin{array}{ccc}
0 & -1 & 0 \\
1 & 0 & 0 \\
0 & 0 & 0
\end{array}\right], \quad K=\left[\begin{array}{ccc}
0 & 0 & -1 \\
0 & 0 & 0 \\
1 & 0 & 0
\end{array}\right]
$$

$M$ and $K$ may be identified with a pair of degenerate 2 -forms $\omega^{(1)}$ and $\omega^{(2)}$ on $P_{\mathscr{L}}$ which define Bridges' multisymplectic structure, and although it may appear that these two 2 -forms provide a distinct structure from that of the 3 -form in $(4 \cdot 5)$, in fact it is just a particular coordinate representation of the intrinsic structure which we have defined.

Proposition 4.2. If $\mathbb{F} \mathscr{L}: J^{1}(Y) \rightarrow P_{\mathscr{L}}$ is a fibre bundle diffeomorphism and $\phi \in \Gamma\left(\pi_{X Y}\right)$, 
then $\tilde{j}^{1}(\phi)$ is a Hamiltonian system for $\mathscr{H}$ if and only if

$$
\left.\left.\frac{\partial}{\partial x^{0}}\right\lrcorner \tilde{j}^{1}(\phi)^{*}\left(d p^{0} \wedge d \phi\right)+\frac{\partial}{\partial x^{1}}\right\lrcorner \tilde{j}^{1}(\phi)^{*}\left(d p^{1} \wedge d \phi\right)=-d H\left(\tilde{j}^{1}(\phi)\right) .
$$

where (4-8) is equivalent to Bridges' equation (4-6).

Proof. Let $U \in T\left(P_{\mathscr{L}}\right)$ be an arbitrary vector field which in coordinates is

so that

$$
U=U_{x^{0}} \frac{\partial}{\partial x^{0}}+U_{x^{1}} \frac{\partial}{\partial x^{1}}+U_{\phi} \frac{\partial}{\partial \phi}+U_{p^{0}} \frac{\partial}{\partial p^{0}}+U_{p^{1}} \frac{\partial}{\partial p^{1}},
$$

$$
\begin{aligned}
U\lrcorner \Omega_{\mathscr{H}}= & -U_{\phi} d p^{0} \wedge d x^{1}+U_{p^{0}} d \phi \wedge d x^{1}-U_{x^{1}} d \phi \wedge d p^{0} \\
& +U_{\phi} d p^{1} \wedge d x^{0}-U_{p^{1}} d \phi \wedge d x^{0}+U_{x^{0}} d \phi \wedge d p^{1} \\
& +\left(\frac{\partial H}{\partial \phi} U_{\phi}+\frac{\partial H}{\partial p^{0}} U_{p^{0}}+\frac{\partial H}{\partial p^{1}} U_{p^{1}}\right) d x^{1} \wedge d x^{0} \\
& -\frac{\partial H}{\partial \phi} U_{x^{1}} d \phi \wedge d x^{0}-\frac{\partial H}{\partial p^{0}} U_{x^{1}} d p^{0} \wedge d x^{0}-\frac{\partial H}{\partial p^{1}} U_{x^{1}} d p^{1} \wedge d x^{0} \\
& +\frac{\partial H}{\partial \phi} U_{x^{0}} d \phi \wedge d x^{1}+\frac{\partial H}{\partial p^{0}} U_{x^{0}} d p^{0} \wedge d x^{1}+\frac{\partial H}{\partial p^{1}} U_{x^{0}} d p^{1} \wedge d x^{1} .
\end{aligned}
$$

Then

$$
\begin{aligned}
\tilde{j}^{1}(\phi)^{*} & \left.(U\lrcorner \Omega_{\mathscr{H}}\right) \\
= & {\left[U_{\phi}\left(\frac{\partial p^{0}}{\partial x^{0}}+\frac{\partial p^{1}}{\partial x^{1}}+\frac{\partial H}{\partial \phi}\right)+U_{p^{0}}\left(-\frac{\partial \phi}{\partial x^{0}}+\frac{\partial H}{\partial p^{0}}\right)+U_{p^{1}}\left(-\frac{\partial \phi}{\partial x^{1}}+\frac{\partial H}{\partial p^{1}}\right)\right.} \\
& +U_{x^{0}}\left(-\frac{\partial \phi}{\partial x^{0}} \frac{\partial p^{1}}{\partial x^{1}}+\frac{\partial \phi}{\partial x^{1}} \frac{\partial p^{1}}{\partial x^{0}}-\frac{\partial H}{\partial \phi} \frac{\partial \phi}{\partial x^{0}}-\frac{\partial H}{\partial p^{0}} \frac{\partial p^{0}}{\partial x^{0}}-\frac{\partial H}{\partial p^{1}} \frac{\partial p^{1}}{\partial x^{0}}\right) \\
& \left.+U_{x^{1}}\left(\frac{\partial \phi}{\partial x^{0}} \frac{\partial p^{0}}{\partial x^{1}}-\frac{\partial \phi}{\partial x^{1}} \frac{\partial p^{0}}{\partial x^{0}}-\frac{\partial H}{\partial \phi} \frac{\partial \phi}{\partial x^{1}}-\frac{\partial H}{\partial p^{0}} \frac{\partial p^{0}}{\partial x^{1}}-\frac{\partial H}{\partial p^{1}} \frac{\partial p^{1}}{\partial x^{1}}\right)\right] d x^{1} \wedge d x^{0},
\end{aligned}
$$

which vanishes if and only if

$$
d H=-\left(\frac{\partial p^{0}}{\partial x^{0}}+\frac{\partial p^{1}}{\partial x^{1}}\right) d \phi+\frac{\partial \phi}{\partial x^{0}} d p^{0}+\frac{\partial \phi}{\partial x^{1}} d p^{1},
$$

and this is precisely a restatement of $(4 \cdot 8)$. To see that $(4 \cdot 8)$ is equivalent to $(4 \cdot 6)$, simply notice that $\partial_{\mu} \tilde{j}^{1}(\phi)=T \tilde{j}^{1}(\phi) . \partial_{\mu}$ and that $\partial_{\mu} \mathscr{Z}$ is the $\pi_{X, P_{\mathscr{L}}}$-vertical component of $\partial_{\mu} \tilde{j}^{1}(\phi)$.

Thus, we have shown that Bridges' formulation is equivalent to our intrinsically defined multihamiltonian system for the nonlinear wave equation defined over one spatial dimension $(n=1)$. The argument, however, is entirely independent of the number of spatial directions and obviously holds when $X=\mathbb{R}^{n+1}$ and $Y=\mathbb{R}^{n+1} \times \mathbb{R}$, in which case our multihamiltonian system may be expressed as

$$
\left.\frac{\partial}{\partial x^{\mu}}\right\lrcorner \tilde{j}^{1}(\phi)^{*}\left(d p^{\mu} \wedge d \phi\right)=-d H,
$$

or, in terms of Bridges' $n+12$-forms $\omega^{(\mu)}$, as

$$
\omega^{(\mu)}\left(\frac{\partial \tilde{j}^{1}(\phi)}{\partial x^{\mu}}, U\right)=-d H\left(\tilde{j}^{1}(\phi)\right) \cdot U \quad \text { for all } \quad U \in T\left(P_{\mathscr{L}}\right) .
$$


More importantly, as we shall show in Section 6 , in the presence of symmetry, we can assemble these $n+1$ distinct 2 -forms $\omega^{(\mu)}$ into our single $(n+2)$-form $\Omega_{\mathscr{H}}$.

\section{Covariant Noether theory}

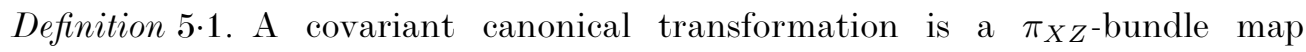
$\eta_{Z}: Z \rightarrow Z$ covering a diffeomorphism $\eta_{X}: X \rightarrow X$ such that $\eta_{Z}^{*} \Omega=\Omega$.

Definition 5.2. If $\eta_{Y}: Y \rightarrow Y$ is a $\pi_{X Y}$-bundle automorphism (also covering a diffeomorphism $\eta_{X}: X \rightarrow X$ ), its canonical lift $\eta_{Z}: Z \rightarrow Z$ is defined by

$$
\eta_{Z}(z)=\left(\eta_{Y}^{-1}\right)^{*}(z) .
$$

We may now define the covariant analogue of momentum maps in symplectic geometry.

Let $\mathscr{G}$ denote a Lie group (perhaps infinite-dimensional) with Lie algebra $\mathfrak{g}$ that acts on $X$ by diffeomorphisms and acts on $Z$ (or $Y$ ) as $\pi_{X Z}$ (or $\pi_{X Y}$ )-bundle automorphisms. For $\eta \in \mathscr{G}$, let $\eta_{X}, \eta_{Y}$ and $\eta_{Z}$ denote the corresponding transformations of $X, Y$ and $Z$ (the map $\eta_{Z}: Z \rightarrow Z$ is the prolongation of $\eta_{Y}$ ) and for $\xi \in \mathfrak{g}$, let $\xi_{X}, \xi_{Y}$ and $\xi_{Z}$ denote the corresponding infinitesimal generators. If $\mathscr{G}$ acts on $Z$ by covariant canonical transformations then the Lie derivative of $\Omega$ along $\xi_{Z}$ is zero:

$$
\mathfrak{S}_{\xi_{Z}} \Omega=0,
$$

so that the left Lie algebra action is canonical. In the case that

$$
\mathfrak{S}_{\xi_{Z}} \Theta=0,
$$

then $\mathscr{G}$ acts by special covariant transformations.

Definition $5 \cdot 3$. Let a Lie algebra $\mathfrak{g}$ have a canonical left action on $Z$ and suppose there exists $J \in L\left(\mathfrak{g}, \Lambda^{n}(Z)\right)$ covering the identity on $Z$ such that for each $\xi \in \mathfrak{g}$,

$$
\left.\xi_{Z}\right\lrcorner \Omega=d J(\xi) .
$$

The map $\mathbf{J}: Z \rightarrow \mathfrak{g}^{*} \otimes \Lambda^{n}(Z)$ defined by

$$
\mathbf{J}(z)(\xi)=J(\xi)(z)
$$

for all $\xi \in \mathfrak{g}$ and $z \in Z$ is called a covariant momentum mapping (or a multimomentum mapping) of the action.

The covariant momentum map is said to be $\mathrm{Ad}^{*}$-equivariant if the diagram

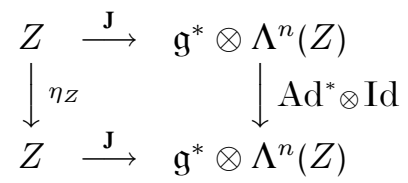

commutes, or equivalently if

$$
J\left(\operatorname{Ad}_{\eta}^{-1} \xi\right)=\eta_{Z}^{*}[J(\xi)] .
$$

Lemma 5.1. If the action on $Z$ is the lifted action $\eta_{Z}$, then $\mathscr{G}$ acts by special covariant transformations, the mapping $J$ defined by

$$
\begin{aligned}
J(\xi) & \left.=\xi_{Z}\right\lrcorner \Theta \\
& \left.=\pi_{Y Z}^{*}\left(\xi_{Y}\right\lrcorner z\right),
\end{aligned}
$$


is a multimomentum mapping of the action for the multisymplectic form on $\Omega$, i.e.

$$
\left.\xi_{Z}\right\lrcorner \Omega=d J(\xi),
$$

and is $\mathrm{Ad}^{*}$-equivariant.

Proof. Differentiating the coordinate expression for $(5 \cdot 1)$ we find that if $\xi=\left(\xi^{\mu}, \xi^{A}\right)$, then

$$
\xi_{Z}=\left(\xi^{\mu}, \xi^{A},-p \xi^{\nu}{ }_{, \nu}-p_{B}{ }^{\nu} \xi^{B}{ }_{, \nu}, p_{A}{ }^{\nu} \xi^{\mu}{ }_{, \nu}-p_{B}{ }^{\mu} \xi^{B}{ }_{, A}-p_{A}{ }^{\mu} \xi^{\nu}{ }_{, \nu}\right),
$$

and hence that $\mathfrak{Q}_{\xi_{z}} \Theta=0$. Then,

$$
\left.\left.\left.d J(\xi)=d\left(\xi_{Z}\right\lrcorner \Theta\right)=\mathfrak{Q}_{\xi_{Z}} \Theta-\xi_{Z}\right\lrcorner d \Theta=\xi_{Z}\right\lrcorner \Omega .
$$

Since $\xi_{Y}=T \pi_{Y Z} \circ \xi_{Z},(5 \cdot 6)$ immediately follows and the last assertion holds because special covariant momentum maps are $\mathrm{Ad}^{*}$-equivariant (the argument is analogous to that for the cotangent bundle case which is proven in Abraham and Marsden [1, theorem $4 \cdot 2 \cdot 10]$ ).

In coordinates this special covariant momentum map may be expressed as

$$
\left.\left.J(\xi)(z)=\left(p_{A}{ }^{\mu} \xi^{A}+p \xi^{\mu}\right) d^{n} x_{\mu}-p_{A}{ }^{\mu} \xi^{\nu} d y^{A} \wedge \partial_{\nu}\right\lrcorner\left(\partial_{\mu}\right\lrcorner d^{n+1} x\right) .
$$

Next we describe the prolonged action of the group $\mathscr{G}$ on $Y$ to $J^{1}(Y)$ and $P_{\mathscr{L}}$.

Definition 5.4. Let $\eta_{Y}: Y \rightarrow Y$ be a $\pi_{X Y}$-bundle automorphism covering a diffeomorphism $\eta_{X}: X \rightarrow X$. Then

$$
\eta_{J^{1}(Y)}(\gamma)=T \eta_{Y} \circ \gamma \circ T \eta_{X}^{-1} \quad \text { for all } \quad \gamma \in J^{1}(Y)
$$

and

$$
\eta_{P_{\mathscr{L}}}(z)=i_{J^{1}(Y)^{\star}, P_{\mathscr{P}}}^{*} \mathbb{F} \mathscr{L}\left(\eta_{J^{1}(Y)} \circ\left(\left.\mathbb{F} \mathscr{L}\right|_{P_{\mathscr{L}}}\right)^{-1} z\right) \quad \text { for all } \quad z \in P_{\mathscr{L}} .
$$

Definition 5.5. We say that the Lagrangian density $\mathscr{L}$ and the Hamiltonian density $\mathscr{H}$ are equivariant with respect to $\mathscr{G}$ if for all $\eta \in \mathscr{G}, \gamma \in J^{1}(Y)$ and $z \in P_{\mathscr{L}}$,

$$
\mathscr{L}\left(\eta_{J^{1}(Y)}(\gamma)\right)=\left(\eta_{X}^{-1}\right)^{*} \mathscr{L}(\gamma),
$$

and

$$
\mathscr{H}\left(\eta_{P_{\mathscr{L}}}(z)\right)=\left(\eta_{X}^{-1}\right)^{*} \mathscr{H}(z)
$$

where $\left(\eta_{X}^{-1}\right)^{*} \mathscr{L}(\gamma)$ means the $(n+1)$-form $\mathscr{L}(\gamma)$ at $x \in X$ is pushed forward to an $(n+1)$-form at $\eta(x)$.

Analogous to [1, corollary $4 \cdot 2 \cdot 14]$, one may readily verify that both $\Theta_{\mathscr{L}}$ and $\Theta_{\mathscr{H}}$ are invariant under the respective group action prolongations, i.e.

$$
\eta_{J^{1}(Y)}^{*} \Theta_{\mathscr{L}}=\Theta_{\mathscr{L}} \quad \text { and } \quad \eta_{P_{\mathscr{L}}}^{*} \Theta_{\mathscr{H}}=\Theta_{\mathscr{H}} \text {. }
$$

Lemma 5.2. Suppose that $\mathscr{L}$ is regular and that $\mathscr{H}: P_{\mathscr{L}} \rightarrow \Lambda^{n+1}(X)$ is equivariant and is not constant on any $P_{\mathscr{L}}$ neighbourhood. Then $\mathscr{L}: J^{1}(Y) \rightarrow \Lambda^{n+1}(X)$ is equivariant.

Proof. Let $\mathscr{G}$ be a group acting on $Y$ by bundle automorphisms. From (5·11), we have by definition that $\mathbb{F} \mathscr{L}: J^{1}(Y) \rightarrow P_{\mathscr{L}}$ is equivariant with respect to $\mathscr{G}$. Hence, for all $\eta \in \mathscr{G}$ and $\left.\gamma \in J^{(} Y\right)$,

$$
\mathscr{H}\left(\eta_{P_{\mathscr{L}}}(\mathbb{F} \mathscr{L}(\gamma))\right)=\left(\eta_{X}^{-1}\right)^{*} \mathscr{H}(\mathbb{F} \mathscr{L}(\gamma)) .
$$


Assume that the Lagrangian density $\mathscr{L}$ is not equivariant with respect to $\mathscr{G}$. Then, there exists $\eta \in \mathscr{G}$ and $\gamma \in J^{1}(Y)$ for which

$$
F(\gamma) \equiv\left[\mathscr{L} \circ \eta_{J^{1}(Y)}(\gamma)-\left(\eta_{X}^{-1}\right)^{*} \mathscr{L}\right](\gamma) \neq 0 .
$$

Hence, by continuity of $F$, there is some neighbourhood $U$ in $J^{1}(Y)$ about $\gamma$ for which $F(U)$ does not intersect $\{0\}$ in $\Lambda^{n+1}(X)$. We will assume that $\gamma \in J^{1}(Y)_{y}$ for some fixed $y \in Y$ and take $U$ to be a fibre neighbourhood of $\gamma$. By choosing $\varepsilon>0$ sufficiently small, we see that for all $\gamma^{\prime} \in J^{1}(Y)_{y}$ satisfying $\mathfrak{A} \circ \gamma^{\prime}=\mathfrak{A}$,

$$
\left.\frac{d}{d \varepsilon}\right|_{\varepsilon=0} \mathscr{L}\left(\eta_{J^{1}(Y)}(\gamma)+\varepsilon\left(\eta_{J^{1}(Y)}\left(\gamma^{\prime}\right)-\eta_{J^{1}(Y)}(\gamma)\right) \neq\left.\left(\eta_{X}^{-1}\right)^{*} \frac{d}{d \varepsilon}\right|_{\varepsilon=0} \mathscr{L}\left(\gamma+\varepsilon\left(\gamma^{\prime}-\gamma\right)\right),\right.
$$

in which case,

$$
D_{\mathfrak{I}}\left(\eta_{J^{1}(Y)}(\gamma)\right) \cdot \eta_{J^{1}(Y)}(\gamma) \neq\left(\eta_{X}^{-1}\right)^{*} D_{\mathscr{U}}(\gamma) \cdot \gamma
$$

for all $\gamma$ in some $J^{1}(Y)$-neighbourhood $U$. Since $\mathscr{H}$ cannot be locally constant (5·14) cannot be true for all $\gamma \in U$ and so $\mathscr{H}$ cannot be equivariant.

Theorem 5-1. Let $\mathscr{L}$ be a regular Lagrangian density and suppose that

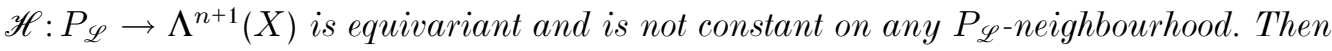
the map

$$
J^{\mathscr{L}}(\xi):=\mathbb{F} \mathscr{L}^{*} J(\xi)
$$

is a momentum map for the lifted action of $\mathscr{G}$ on $J^{1}(Y)$ relative to $\Omega_{\mathscr{L}}$ and the map

$$
J^{\mathscr{H}}(\xi):=\left(\left(\left.\mathbb{F} \mathscr{L}\right|_{P_{\mathscr{L}}}\right)^{-1}\right)^{*} \circ \mathbb{F} \mathscr{L}^{*} J(\xi)=\left(\left.\mathbb{F} \mathscr{L} \circ \mathbb{F} \mathscr{L}\right|_{P_{\mathscr{L}}} ^{-1}\right)^{*} J(\xi)=i_{J^{1}(Y)^{\star}, P_{\mathscr{L}}}^{*} J(\xi)
$$

is a momentum map for the lifted action of $\mathscr{G}$ on $P_{\mathscr{L}}$ relative to $\Omega_{\mathscr{H}}$, i.e. for all $\xi \in \mathfrak{g}$,

$$
\left.\xi_{J^{1}(Y)}\right\lrcorner \Omega_{\mathscr{L}}=d J^{\mathscr{L}}(\xi)
$$

and

$$
\left.\xi_{P_{\mathscr{L}}}\right\lrcorner \Omega_{\mathscr{H}}=d J^{\mathscr{H}}(\xi),
$$

where $\xi_{J^{1}(Y)}$ and where $\xi_{P_{\mathscr{P}}}$ are the infinitesimal generators corresponding to $\xi$.

Proof. Lemma $5 \cdot 2$ asserts that $\mathscr{L}$ is equivariant, from which we may conclude that $\mathbb{F} \mathscr{L}: J^{1}(Y) \rightarrow Z$ is equivariant so that

$$
\eta_{Z} \circ \mathbb{F} \mathscr{L}=\mathbb{F} \mathscr{L} \circ \eta_{J^{1}(Y)} .
$$

Indeed, we see that

$$
\begin{aligned}
\left\{\eta_{J^{1}(Y)^{*}}[\mathbb{F} \mathscr{L}(\gamma)]\right\} \cdot \gamma^{\prime} & =\left(\eta_{X}^{-1}\right)^{*}\left\{\mathbb{F} \mathscr{L}(\gamma)\left[\eta_{J^{1}(Y)}^{-1}\left(\gamma^{\prime}\right)\right]\right\} \\
& =\left(\eta_{X}^{-1}\right)^{*}\left\{\mathscr{L}(\gamma)+\left.\frac{d}{d \varepsilon}\right|_{\varepsilon=0} \mathscr{L}\left(\gamma+\varepsilon\left[\eta_{J^{1}(Y)}^{-1}\left(\gamma^{\prime}\right)-\gamma\right]\right)\right\} .
\end{aligned}
$$

and that

$$
\left.\left\{\mathbb{F} \mathscr{L}\left[\eta_{J^{1}(Y)}(\gamma)\right]\right\} \cdot \gamma^{\prime}=\mathscr{L}\left(\eta_{J^{1}(Y)}(\gamma)\right)+\left.\frac{d}{d \varepsilon}\right|_{\varepsilon=0} \mathscr{L}\left(\eta_{J^{1}(Y)}(\gamma)\right)+\varepsilon\left[\gamma^{\prime}-\eta_{J^{1}(Y)}(\gamma)\right]\right),
$$

which are equal by the equivariance of $\mathscr{L}$. The infinitesimal version of $(5 \cdot 17)$ yields

$$
\xi_{Z} \circ \mathbb{F} \mathscr{L}=T \mathbb{F} \mathscr{L} \cdot \xi_{J^{1}(Y)},
$$


which is a statement that $\xi_{Z}$ is $\mathbb{F} \mathscr{L}$-related to $\xi_{J^{1}(Y)}$. Hence, the pull-back of (5·7) along $\mathbb{F} \mathscr{L}$ gives us (5·15), while a second pull-back of $(5 \cdot 15)$ along the diffeomorphism $\left.\mathbb{F} \mathscr{L}\right|_{P_{\mathscr{P}}} ^{-1}$ verifies $(5 \cdot 16)$.

In multisymplectic coordinates, the multimomentum mapping $J^{\mathscr{H}}$ is written as

$$
\left.\left.J^{\mathscr{H}}(\xi)=\left(p_{A}{ }^{\mu} \xi^{A}+\left(p_{A}{ }^{\nu} \mathfrak{A}_{\nu}^{A}-H\right) \xi^{\mu}\right) d^{n} x_{\mu}-p_{A}{ }^{\mu} \xi^{\nu} d y^{A} \wedge \partial_{\nu}\right\lrcorner\left(\partial_{\mu}\right\lrcorner d^{n+1} x\right),
$$

where $H: P_{\mathscr{L}} \rightarrow \mathbb{R}$ is the Hamiltonian function associated with the Hamiltonian density $\mathscr{H}$ by $\mathscr{H}=H d^{n+1} x$.

Theorem $5 \cdot 2$ (Covariant Hamiltonian version of Noether's theorem). Let $\mathscr{L}$ be regular and suppose that a group $\mathscr{G}$ acts on $Y$ by $\pi_{X Y}$-bundle automorphisms, and that the Hamiltonian density $\mathscr{H}$ is equivariant with respect to $\mathscr{G}$ and is not locally constant

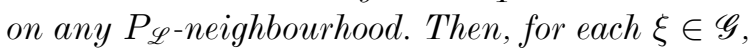

$$
d\left[\tilde{j}^{1}(\phi)^{*} J^{\mathscr{H}}(\xi)\right]=0,
$$

for any $\phi \in \Gamma\left(\pi_{X Y}\right)$ for which $\tilde{j}^{1}(\phi)$ is a covariant Hamiltonian system.

Proof. Since $\tilde{j}^{1}(\phi)$ is a Hamiltonian system, $\tilde{j}^{1}(\phi)^{*} i_{U} \Omega_{\mathscr{H}}=0$ for any vector $U$ in $T\left(P_{\mathscr{L}}\right)$ so set $U=\xi_{P_{\mathscr{P}}}$.

Under the same hypotheses, Lemma $3 \cdot 1$ gives us the following equivalent statement.

Corollary 5·1. For each $\xi \in \mathscr{G}, d\left[j^{1}(\phi)^{*} J^{\mathscr{L}}(\xi)\right]=0$.

The quantity $\tilde{j}^{1}(\phi)^{*} J^{\mathscr{H}}(\xi)$ is called the Hamiltonian Noether current and, as we shall show, leads to very useful decompositions of the classical water wave conservations laws. In coordinates, it has the form

$$
\tilde{j}^{1}(\phi)^{*} J^{\mathscr{H}}(\xi)=\left[\left(p_{A}{ }^{\mu} \xi^{A}+\left(p_{A}{ }^{\nu} \mathfrak{Q}_{\nu}^{A}-H\right) \xi^{\mu}\right)-p_{A}{ }^{\mu} \phi^{A}{ }_{, \nu} \xi^{\nu}+p_{A}{ }^{\nu} \phi_{, \nu}^{A} \xi^{\mu}\right] d^{n} x_{\mu} .
$$

\section{Symmetry and generalized conservation laws}

Just as we have shown in Section 4 that the multihamiltonian system generalizes the classical Hamiltonian description of particle mechanics as well as the structures defined in Bridges [4], we can do the same for the multisymplectic Hamiltonian Noether theory.

\subsection{Particle mechanics}

We let the groups Diff $(\mathbb{R})$ act on $\mathbb{R}$ and $G$ on $Q$ and consider the action of the prolongation of $\mathscr{G}=\operatorname{Diff}(\mathbb{R}) \times G$. With elements of $\mathfrak{g}$ written as $(f, \xi)$, the Hamiltonian Noether current has the simple coordinate form

$$
\tilde{j}^{1}(\phi)^{*} J^{\mathscr{H}}(\xi)=p_{A} \xi^{A}-H f:=J_{\xi}^{H}-H f,
$$

where $J_{\xi}^{H}=p_{A} \xi^{A}$ is the usual momentum map for $G$ acting on $Q$ in Hamiltonian mechanics. Then equation $(5 \cdot 21)$ asserts that along trajectories of the Hamiltonian vector field

$$
\frac{d}{d t}\left(J_{\xi}^{H}-H f\right)=0
$$

which is equivalent to conservation of both $J_{\xi}^{H}$ and $H$. 


\subsection{Bridges' decomposition of Noether theory}

In this section we fix $X=\mathbb{R}^{n+1} Y=\mathbb{R}^{n+1} \times \mathbb{R}$ and $P_{\mathscr{L}}=\pi_{\mathbb{R}^{n+1}, \mathbb{R}^{n+2}}$. We examined this particular geometry in Section $4 \cdot 2$, where we showed that Bridges' multisymplectic structure consisting of $n+1$ degenerate 2 -forms $\omega^{(\mu)}$ on $P_{\mathscr{L}}$ is an equivalent and particular coordinate representation of our intrinsically defined multihamiltonian system on $P_{\mathscr{L}}$. We now show that in the presence of a general symmetry group action, Bridges' $n+1$ pre-symplectic 2 -forms $\omega^{(\mu)}$ can actually be assembled into our single multisymplectic $(n+2)$-form $\Omega^{\mathscr{H}}$.

Bridges forms an $(n+1)$-vector of momentum mappings such that each vector component is associated with a distinct spacetime direction through the pre-symplectic 2 -form $\omega^{(\mu)}$. He then relates the action of a general symmetry group $\mathscr{G}$ along the fibre of $P_{\mathscr{L}}$ with the vanishing of the divergence of the vector of momentum mappings. The following result is in $[2$, theorem $2 \cdot 2]$.

Proposition 6 1 . Let $H: \mathbb{R}^{n+2} \rightarrow \mathbb{R}$ be a covariant Hamiltonian with $n+1$ distinct 2 -forms $\omega^{(\mu)}$. If $d H \cdot \xi_{P_{\mathscr{P}}}=0$ for all $\xi_{P_{\mathscr{P}}}$ in the Lie algebra $\mathfrak{g}$ of the group $\mathscr{G}$ acting on $\mathbb{R}^{n+2}$, and if $P^{\mu}$ is the momentum mapping associated to $\omega^{(\mu)}$, i.e. for all $\xi_{P_{\mathscr{P}}} \in \mathfrak{g}$,

$$
\left.\xi_{P_{\mathscr{L}}}\right\lrcorner \omega^{(\mu)}=d P^{\mu}\left(\xi_{P_{\mathscr{Q}}}\right),
$$

then

$$
\frac{\partial P^{\mu}}{\partial x^{\mu}}=0 .
$$

We will first show that $(6 \cdot 2)$ is a particular example of our conservation law $(5 \cdot 21)$ in the case of the trivial bundle geometry defined above, for which the fields have no explicit dependence on time or space (geometrically, this means that each fiber of the bundle $\pi_{X, P_{\mathscr{P}}}$ is identical).

Proposition $6 \cdot 2$. In the case that the action on $P_{\mathscr{L}}:=\pi_{\mathbb{R}^{n+1}, \mathbb{R}^{n+2}}$ is the lifted action $\eta_{P_{\mathscr{q}}}$, then the momentum mappings $P^{\mu}$ defined in (6-1) are the components of the Hamiltonian Noether current and hence the conservation law (6.2) is contained in Theorem 5.2.

Proof. We set the diffeomorphism $\eta_{X}$ to be the identity and identify $P_{\mathscr{L}}$ with $\mathbb{R}^{n+2}$. Hence the infinitesimal generators $\xi^{\mu}$ are 0 and, using $(5 \cdot 22)$, we see that the Hamiltonian Noether current is given by

$$
\tilde{j}^{1}(\phi)^{*} J^{\mathscr{H}}(\xi)=p^{\mu} \xi d^{n} x_{\mu}:=N^{\mu} d^{n} x_{\mu} .
$$

Using (5.8), we easily deduce that the lifted action $\xi_{P_{\mathscr{T}}}$ is given in coordinates by $\left(0, \xi,-p^{\mu} \partial \xi / \partial \phi\right)$ so that the equivariance of $\mathscr{H}$ is equivalent to $d H . \xi_{P_{\mathscr{L}}}=0$.

In accordance with Proposition $6 \cdot 1$, all of the group action is along the fibre of $\pi_{X, P_{\mathscr{P}}}$, identified with $\mathbb{R}^{n+2}$, so we will restrict the exterior derivative $d$ to the fibre.

We claim that $P^{\mu}=N^{\mu}$. To see this we must show that $\left.d N^{\mu}=\xi_{P_{\mathscr{P}}}\right\lrcorner \omega^{(\mu)}$, but this is precisely the case since in coordinates, for each $\mu=1, \ldots, n, 0$,

$$
d N^{\mu}=\left(p^{\mu} \frac{\partial \xi}{\partial \phi}, 0, \ldots, \xi, \ldots, 0\right), \quad \xi \text { in the }(\mu+1) \text { th coordinate. }
$$

Then, using the identity

$$
d\left(N^{\mu} d^{n} x_{\mu}\right)=\partial_{\nu} N^{\mu} d x^{\nu} \wedge d^{n} x_{\mu}=\partial_{\mu} N^{\mu} d^{n+1} x
$$


we have that $d\left[\tilde{j}^{1}(\phi)^{*} J^{\mathscr{H}}(\xi)\right]=0$ implies that $\partial N^{\mu} / \partial x^{\mu}=0$ so that $\partial P^{\mu} / \partial x^{\mu}=0$ and the result is proved.

This proposition indicates how we can assemble the 2 -forms $\omega^{(\mu)}$ into the single $(n+2)$-form $\Omega_{\mathscr{H}}$ when lifted symmetries exist. Namely, to each $\omega^{(\mu)}$, there corresponds a momentum mapping $P^{\mu}\left(\xi_{P_{\mathcal{P}}}\right)$ of the symmetry group given by $(6 \cdot 1)$. By Proposition 6.2, the maps $P^{\mu}\left(\xi_{P_{\mathscr{L}}}\right)$ are the components of the Hamiltonian Noether current. This then defines the Hamiltonian covariant momentum mapping $J^{\mathscr{H}}\left(\xi_{P_{\mathscr{\varphi}}}\right)$ which in turn, by $(5 \cdot 16)$, defines the canonical multisymplectic $(n+2)$-form $\Omega_{\mathscr{H}}$ on $P_{\mathscr{L}}$. In fact, since lifts are special canonical transformations, the covariant momentum map defines the $(n+1)-\Theta_{\mathscr{H}}$ on $P_{\mathscr{L}}$ as well. We summarize with the following corollary.

Corollary 6.1. Assume the group $\mathscr{G}$ acts by special canonical transformations and let the Hamiltonian Noether current $\tilde{j}^{1}(\phi)^{*} J^{\mathscr{H}}(\xi)$ be given in multisymplectic coordinates by $N^{\mu} d^{n} x_{\mu}$. Then the $\omega^{(\mu)}$ satisfy $\left.\xi_{P \mathscr{P}}\right\lrcorner \omega^{(\mu)}=d N^{\mu}$. Furthermore, if $\omega^{(\mu)}$ is exact, such that

$$
\omega^{(\mu)}=d \kappa^{(\mu)}
$$

for 1 -forms $\kappa^{(\mu)}$, then

$$
\left.N^{\mu}=\xi_{P \mathscr{L}}\right\lrcorner \kappa^{(\mu)}
$$

\section{The Geometry of water waves}

It is interesting to note that the covariant Hamiltonian Noether theorem intrinsically contains the mass conservation law for water waves as well as the conservation of wave action and action flux. In particular, the vanishing of the exterior derivative of the Hamiltonian Noether current is an intrinsic restatement of the mass conservation law, while the projected components of the Hamiltonian Noether current $P^{\mu}$, as defined by Proposition $6 \cdot 2$, are related to the action and action flux. As an example, for the case of two spatial dimensions, the ensemble (or phase) average of $P^{0}$ corresponds to Whitham's definition of wave action and that of $P^{1}$ and $P^{2}$ correspond to the two-component action-flux (see [10]), while in the case of one spatial dimension the Hamiltonian density $\mathscr{H}$ is related to the flow force or in some cases the momentum flux (see [3]). These observations were first made by Bridges [2] in coordinates; they seemed to have been the primary motivating factors for defining additional 2 -forms $\omega^{(\mu)}$ for each unbounded spatial direction.

Next, we show that our definition for a multihamiltonian system contains the variational principles which are essential to the study of pattern formation and wave instability. For simplicity, we restrict our attention to symmetries given by the circle and $\mathbb{T}^{n+1}$; however, it is important to note that our procedure is general and may be applied to any subgroup of the Euclidean group SE $(n+1)$ and its products. This is significant if one wishes to study hexagonal pattern formations, for example, in addition to merely the periodic ones.

\section{7·1. Pattern formation, action, index and the loop space}

Let $X=\mathbb{R}^{n+1}$ and let $Y$ be the vector bundle $\mathbb{R}$ over $X$. Consider the semilinear elliptic scalar partial differential equation

$$
\triangle \phi+V^{\prime}(\phi)=0, \quad \phi \in \Gamma\left(\pi_{X Y}\right),
$$


where, as above, $\triangle$ is the Laplace-Beltrami operator and $V$ is a real-valued $C^{\infty}$-bundle map. For this example, it is appropriate to set $P_{\mathscr{L}}=\pi_{\mathbb{R}^{n+1}, \mathbb{R}^{n+2}}$ and $\mathscr{G}=\mathrm{SO}(n+1)$, in which case $(7 \cdot 1)$ may be equivalently expressed as

$$
\left.\tilde{j}^{1}(\phi)^{*}(U\lrcorner \Omega_{\mathscr{C}}\right)=0
$$

for all $U \in T\left(P_{\mathscr{L}}\right)$, where in coordinates,

$$
\mathscr{H}=H d^{n+1} x \quad \text { and } \quad H\left(\tilde{j}^{1}(\phi)\right)=\frac{1}{2} p^{\mu} \cdot p^{\mu}+V(\phi) .
$$

We show in this section that our intrinsic multisymplectic structure can be used to generalize the notion of action and index on the loop space of the primary constraint subbundle $P_{\mathscr{L}}$ as defined in [3].

Let the map $\chi: X \rightarrow \mathbb{R}$ be defined in coordinates by $\chi\left(x^{\mu}\right)=k_{\mu} x^{\mu}$, and identify $\mathbb{T}^{1}$ with its universal cover $\mathbb{R} \backslash \mathbb{Z}$, so that a smooth $2 \pi$-periodic map $\alpha: \mathbb{R} \rightarrow P_{\mathscr{L}}$ may be identified with the smooth map $\alpha: \mathbb{T}^{1} \rightarrow P_{\mathscr{L}}$.

Definition $7 \cdot 1$. The loop space of $P_{\mathscr{L}}$ is the subset of $\Gamma\left(\pi_{X, P_{\mathscr{L}}}\right)$ defined by

$$
\operatorname{Loop}\left(P_{\mathscr{L}}\right)=\left\{z \in \Gamma\left(\pi_{X, P_{\mathscr{P}}}\right) \mid z=\alpha \circ \chi \text { is holonomic and } \alpha \in C^{\infty}\left(\mathbb{T}^{1}, P_{\mathscr{L}}\right)\right\} \text {. }
$$

We then set

$$
\operatorname{loop}\left(P_{\mathscr{L}}\right)=\left\{z \in \operatorname{Loop}\left(P_{\mathscr{L}}\right) \mid \dot{\alpha} \in \mathscr{P}\right\},
$$

where $\dot{\alpha}=d \alpha / d \chi$ and $\mathscr{P} \subset T\left(P_{\mathscr{L}}\right)$ consists of those vector fields on $P_{\mathscr{L}}$ which are prolongations of vector fields on $Y$.

Hence, an element $\alpha \circ \chi$ in $\operatorname{loop}\left(P_{\mathscr{L}}\right)$ is conjugate to the first jet of a section $f \circ \chi$ in $\pi_{X Y}$, where $f: \mathbb{T}^{1} \rightarrow Y$.

The diagonal periodic patterns of $(7 \cdot 1)$ correspond to the restriction of $(7 \cdot 1)$ to Loop $\left(P_{\mathscr{L}}\right)$. Thus, if $\alpha \circ \chi \in \operatorname{Loop}\left(P_{\mathscr{L}}\right)$, a diagonal periodic pattern satisfies

$$
\left.(\alpha \circ \chi)^{*}(U\lrcorner \Omega_{\mathscr{H}}\right)=0 \text { for all } U \in T\left(P_{\mathscr{L}}\right) .
$$

Recently, the existence of periodic pattern solutions to $(7 \cdot 1)$ has been obtained by expressing such solutions as critical points of a constrained variational principle and using information provided by sensitivity matrices, sometimes called the index, for classification of the critical point type. As it turns out, when the infinitesimal group action coincides with the vector field $\dot{\alpha}$, the Hamiltonian Noether current naturally and intrinsically verifies these variational principles.

When $\alpha \circ \chi \in \operatorname{loop}\left(P_{\mathscr{L}}\right)$, we may associate to it the loop space Hamiltonian Noether current

$$
\mathscr{N}(\dot{\alpha}):=(\alpha \circ \chi)^{*} J^{\mathscr{H}}(\dot{\alpha}) .
$$

As with our previous example in Section $4 \cdot 2$, we set the group action on $X$ to be the identity and identify $P_{\mathscr{L}}$ with $\mathbb{R}^{n+2}$. We assume that $\mathscr{H}$ is equivariant with respect to the group action on $P_{\mathscr{L}}$ and is not locally constant. In this case, $d \mathscr{N}(\dot{\alpha})=0$ implies that $\alpha \circ \chi:=\tilde{j}^{1}(f \circ \chi)$ is a Hamiltonian system, a fact that is readily verified by a tedious computation in local coordinates. The computation, however, proceeds easily on the Lagrangian side. Using the coordinate expression

$$
\frac{d}{d \chi} j^{1}(f \circ \chi)=j^{1}(\dot{f}):=\left(0, \dot{f}, \frac{\partial \dot{f}}{\partial x^{\mu}}+\frac{\partial \dot{f}}{\partial y} \frac{\partial(f \circ \chi)}{\partial x^{\mu}}\right),
$$


which is readily obtained from the definition of the vector field prolongation to $J^{1}(Y)$ given in (5·10), we appeal to Corollary $5 \cdot 1$ and check that $d\left[j^{1}(f \circ \chi)^{*} J^{\mathscr{L}}\left(j^{1}(\dot{f})\right)\right]=0$. In coordinates, this yields

$$
\begin{aligned}
& \left\{\left[\frac{\partial L}{\partial y}\left(j^{1}(f \circ \chi)\right)-\frac{\partial}{\partial x^{\mu}}\left(\frac{\partial L}{\partial(f \circ \chi)_{\mu}}\left(j^{1}(f \circ \chi)\right)\right)\right][-\dot{f} \circ(f \circ \chi)]\right. \\
& \quad+\frac{\partial L}{\partial y}\left(j^{1}(f \circ \chi)\right)(\dot{f} \circ(f \circ \chi)) \\
& \left.\quad+\frac{\partial L}{\partial(f \circ \chi)_{\mu}}\left(j^{1}(f \circ \chi)\right)\left[\frac{\partial \dot{f}}{\partial x^{\mu}}+\frac{\partial \dot{f}}{\partial y}(f \circ \chi)_{\mu}\right]\left(j^{1}(f \circ \chi)\right)\right\} d^{n+1} x
\end{aligned}
$$

which vanishes if and only if the Lagrangian is equivariant and if $f \circ \chi$ is a stationary point of $\int_{X} \mathscr{L}\left(j^{1}(f \circ \chi)\right)$. Since $\mathscr{H}$ is equivariant, Lemma $5 \cdot 2$ guarantees that $\mathscr{L}$ is equivariant as well, and Theorem $3 \cdot 1$ gives us that $\alpha \circ \chi$ is a Hamiltonian system.

To see that our covariant Noether theory contains the classical constrained variational principle in local coordinates, we make the following observations. Let the 2 -forms $\omega^{(\mu)}$ and the 1 -forms $\kappa^{(\mu)}$ be as defined in Corollary $6 \cdot 1$. By Proposition $6 \cdot 1$, $(7 \cdot 4)$ is satisfied if and only if

$$
\begin{aligned}
0 & \left.=-d H(\alpha \circ \chi)-k_{\mu}(\dot{\alpha}\lrcorner \omega^{(\mu)}\right) \\
& \left.=d\left[-H(\alpha \circ \chi)-k_{\mu}(\dot{\alpha}\lrcorner \kappa^{(\mu)}\right)\right] \\
& :=d \mathscr{F}(\dot{\alpha}, \chi) .
\end{aligned}
$$

Thus, as noted in [3], $\alpha \circ \chi$ is a diagonal periodic pattern if it is the critical point of $\int_{\mathbb{T}^{1}} \mathscr{F}(\dot{\alpha}, \chi) d \chi$ (classically, the phase-averaged quantities are considered). From this, we see that the solutions to $(7 \cdot 4)$ are the critical points of the phase-averaged Hamiltonian with the additional constraints

$$
\left.\int_{\mathbb{T}^{1}}[\dot{\alpha}\lrcorner \kappa^{(\mu)}\right] d \chi=I_{\mu}
$$

so that the $k_{\mu}$ are the Lagrange multipliers. In the case that $H$ may be viewed as an implicit function of the $I_{\mu}$, we have that

$$
k_{\mu}=-\frac{\partial H}{\partial I_{\mu}},
$$

so that the Hessian matrix of $\mathrm{H}$ with respect to the level sets $I_{\mu}$ has components

$$
\left[\operatorname{Hess}_{I}(H)\right]_{\mu \nu}=\frac{\partial^{2} H}{\partial I_{\mu} \partial I_{\nu}}=\frac{\partial k_{\mu}}{\partial I_{\nu}} .
$$

From the implicit function theorem, a diagonal periodic pattern is non-degenerate if $\operatorname{det}\left[\operatorname{Hess}_{I}(H)\right] \neq 0$. Then the natural definition for the index for such patterns is given by

$$
\text { index }(\alpha)=\# \text { negative eigenvalues of } \operatorname{Hess}_{I}(H) \text {. }
$$

We refer the reader to [2] for a detailed account and applications.

\subsection{Stability of water waves}

Conservative partial differential equations are often accurate models for water waves, and in this section we will briefly comment upon the connection between our 
covariant Noether theory and the constrained toral variational principles which lead to characterizations of the instabilities of the system. Our brevity is due to the fact that the $\operatorname{Hess}_{I}(H)$-matrix is explicitly connected to the linear stability exponents from which we may deduce the behaviour of our solutions and, as we gave a fairly detailed description in the previous section of how this matrix arises from the vanishing of the exterior derivative of the Hamiltonian Noether current, we shall herein only discuss the minor modifications necessary for this theory.

To demonstrate the main ideas, let us consider the the manifolds $X, Y$ and $P_{\mathscr{L}}$ as given in the previous section and the the partial differential equation defined in $(4 \cdot 4)$, with corresponding covariant Hamiltonian

$$
\mathscr{H}=\left[\frac{1}{2}\left(\sum_{\mu=1}^{n} p_{\mu}^{2}-p_{0}^{2}\right)-V(\phi)\right] d^{n+1} x .
$$

Unlike the case of pattern formation for which we considered solutions of

$$
\left.\tilde{j}^{1}(\phi)^{*}(U\lrcorner \Omega_{\mathscr{H}}\right)=0 \quad \text { for all } \quad U \in T\left(P_{\mathscr{L}}\right)
$$

restricted to loop $\left(P_{\mathscr{L}}\right)$, now we restrict consideration to the periodic sections of $P_{\mathscr{L}}$, so that $\tilde{j}^{1}(\phi): \mathbb{T}^{n+1} \rightarrow P_{\mathscr{L}}$. If we make the change of variables $w^{\mu}=k_{\mu} x^{\mu}$ (no sum), then by Proposition $6 \cdot 1$, periodic solutions of $(7 \cdot 7)$ are expressed in coordinates by

$$
\left.k_{\mu} \frac{\partial \tilde{j}^{1}(\phi)}{\partial w^{\mu}}\right\lrcorner \omega^{(\mu)}=-d H\left(\tilde{j}^{1}(\phi)\right), \quad\left(k_{0}, \ldots, k_{n}\right) \in \mathbb{T}^{n+1} .
$$

Arguing exactly as we did in the previous section, we may again deduce that the $k_{\mu}$ are the Lagrange multipliers of the system and thus the Hessian matrix of $H$ with respect to the level set $I_{\mu}$ is identically obtained as for the case of periodic pattern formation. See [4] for a discussion of the relationship between $\operatorname{Hess}_{I}(H)$ and the classical linear stability exponents.

Acknowledgements. The authors would like to thank Tom Bridges and Mark Gotay for reading early drafts of the manuscript and making numerous comments and suggestions. S. S. was partly supported by a fellowship from the Cecil H. and Ida M. Green Foundation. J. E. M. was partly supported by the National Science Foundation under Grant DMS-DMS-96 33161 and the Department of Energy under Contract DE-FG0395-ER25251.

\section{REFERENCES}

[1] R. Abraham and J. Marsden. Foundations of mechanics, Second Edition (Addison-Wesley 1978).

[2] T. J. Bridgess. Periodic patterns, linear instability, symplectic structure and mean-flow dynamics for three-dimensional surface waves. Phil. Trans. R. Soc. Lond. A, 354 (1996), $533-574$.

[3] T. J. Bridges. Symplecity, reversibility and elliptic operators. Progress in Nonlinear Diff. Eq., 19 (1996), 1-20.

[4] T. J. Bridges. Multi-symplectic structures and wave propagation. Math. Proc. Camb. Phil. Soc. 121 (1996), 147-190.

[5] T. J. BRidges. Geometric formulation of the conservation of wave action and its implications for signature and the classification of instabilities. Proc. R. Soc. Lond. A (in press).

[6] F. Cantrijn, L. A. Ibort and M. De León. Hamiltonian structures on multisymplectic manifolds. Rend. Sem. Mat. Torino (in press). 
[7] M. J. Gotay. A multisymplectic framework for classical field theory and the calculus of variations I: Covariant Hamiltonian formulation, in Mechanics, analysis and geometry: 200 Years After Lagrange, M. Francaviglia, Ed., 203-235 (North Holland, 1991).

[8] M. Gotay, J. Isenberg, J. E. Marsden and R. Montgomery. Momentum maps and the Hamiltonian structure of classical relativistic field theories. (unpublished).

[9] M. J. Gotay and J. E. Marsden. Stress-energy-momentum tensors and the BelifanteResenfeld formula. Cont. Math. AMS. 132 (1992), 367-392.

[10] G. B. Wнітнам. Linear and nonlinear waves (Wiley-Interscience, 1974). 